\title{
Relation longueur-poids chez quatre espèces de poissons de la rivière Sourou au Burkina Faso
}

\author{
Nessan Désiré COULIBALY \\ Institut de l'Environnement et de Recherches Agricoles (INERA), 03 BP 7047, Ouagadougou 03, Burkina \\ Faso. Tél. : (00226)33.40.98, Fax :(00226)31.50.03 - E-mail : dnessan@yahoo.fr
}

\section{RESUME}

La relation longueur-poids a été étudiée chez Heterotis niloticus, Gymnarchus niloticus, Protopterus annectens annectens et Clarias gariepinus dans la rivière Sourou à partir des spécimen collectés entre septembre 2003 et novembre 2004. La valeur de « $b »$ a varié de 2,70 à 3,15. Les résultats de cette étude constituent les références disponibles sur les quatre espèces de poissons les plus abondantes et d'intérêt commercial dans la rivière Sourou.

(C) 2008 International Formulae Group. All rights reserved.

Mot-clés: Poisson, longueur-poids, pêcherie continentale, rivière Sourou, Burkina Faso.

\section{INTRODUCTION}

Chez les poissons, la croissance se manifeste par des variations de poids et de longueur et il existe par ailleurs une relation étroite entre ces deux variables (Le Cren, 1951 ; Baijot et al., 1994 ; Pauly et Moreau, 1997 ; Lévêque, 1999). La connaissance de cette relation trouve des applications en biologie des pêches et dans l'évaluation des stocks halieutiques (Kochzius, 1997 ; RuizRamirez, 1997 ; Le Tourneur, 1998 ; Frota et al., 2004). La connaissance des paramètres « a et $\mathrm{b} »$ de la relation longueur-poids, particulièrement la valeur de $« b »$, trouve de nombreuses applications en biologie des pêches. Selon Stergiou et Moutopoulos (2001), les données de la relation longueurpoids sont utiles pour l'estimation de la biomasse à partir des longueurs, l'estimation de la condition (état de bien-être) des populations piscicoles dans un milieu donné, et à la prédiction du poids à partir des longueurs dans l'évaluation des captures. Enfin, les paramètres de la relation constituent un outil essentiel de comparaison entre diverses populations d'une même espèce vivant dans des écosystèmes similaires ou différents (Stergiou et Moutopoulos, 2001;
Thomas et al., 2003; Odat, 2003). Cette étude de la relation longueur-poids a été conduite sur quelques espèces de poissons sur plusieurs plans d'eau douce en Afrique et au Burkina Faso (Tableau 1). De toutes les études réalisées au Burkina Faso sur la biologie, aucune information n'est disponible chez les poissons de la rivière Sourou dans la région Nord-Ouest du Burkina Faso.

Cette rivière du Sourou (figure 1), long de $150 \mathrm{~km}$, est partagée entre le Mali en amont $(90 \mathrm{~km})$ et le Burkina Faso en aval (60 $\mathrm{km})$. Au Burkina Faso, elle a subi deux aménagements hydrauliques qui ont consisté en la construction d'un barrage vanne à sa confluence avec le fleuve Mouhoun en 1976 et au creusement d'un canal reliant directement le Mouhoun au Sourou en 1984 (Baijot et al., 1994b). Le plan d'eau engendré par cet aménagement occupe une superficie inondée de 10.000 ha environ (Baijot et al., 1994b) avec d'immenses prairies aquatiques constituées d'association de Oryza barthii, d'Echinochloa stagnina, de Potamogeton sp ou de Polygonum lanigerum, (Baijot et al.,1994a) favorables à la vie et au développement des poissons. 
Tableau 1: Valeurs des constantes $\mathbf{a}$ et $\mathbf{b}$ des relations entre les longueurs et poids chez quelques espèces de poissons d'eau douce en Afrique.

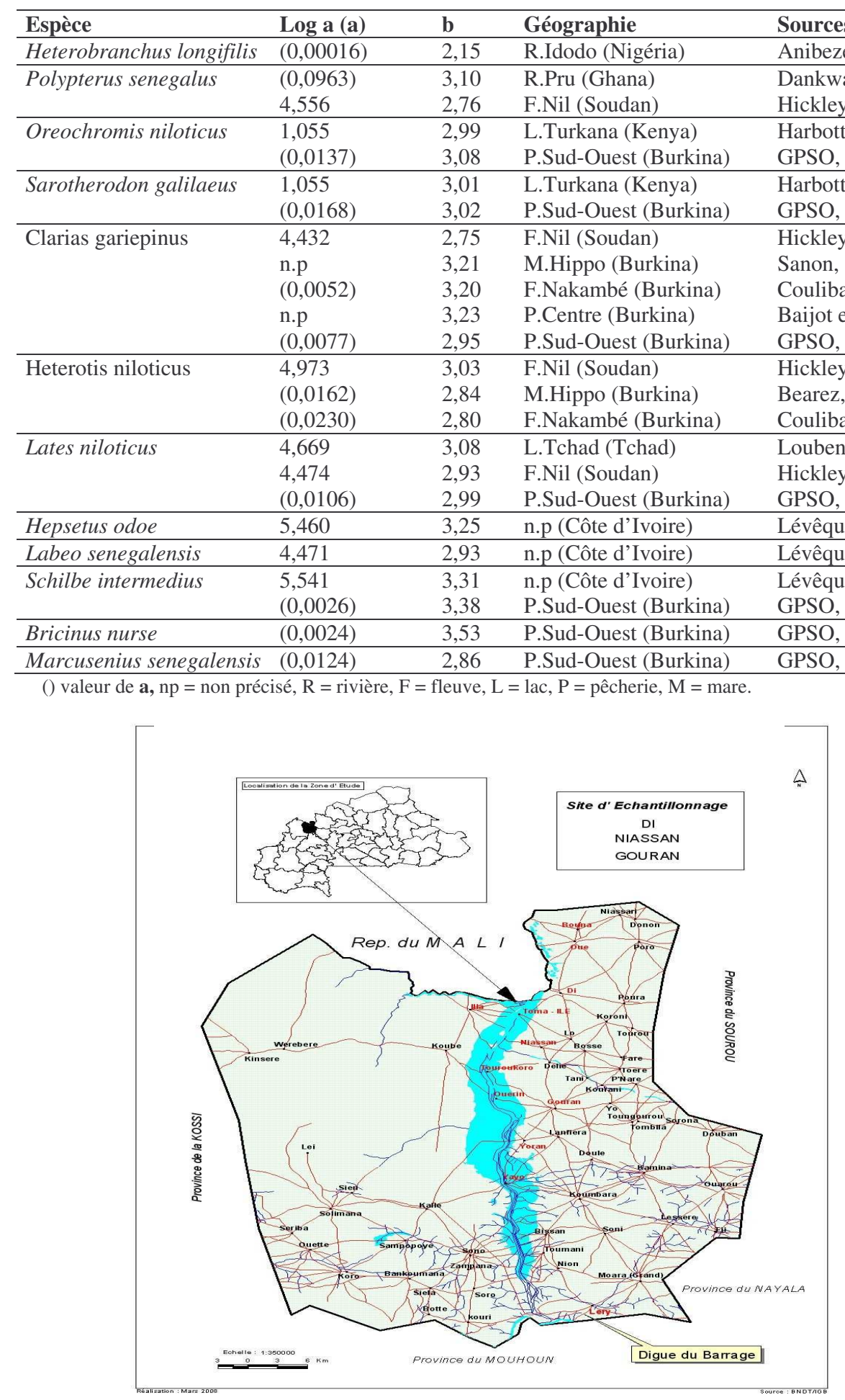

Figure 1: Rivière Sourou au Burkina avec les plaines d'inondation et les sites d'échantillonnage. 
La pêcherie du Sourou occupe le $3^{\text {ème }}$ rang national de la production piscicole après les pêcheries des lacs de Bagré et de Kompienga (Coulibaly, 2006). Le potentiel productif de cette pêcherie est estimé entre 600 et 1000 tonnes/an (Baijot et al., 1994b) contre une production exploitée d'environ 300 tonnes. Au plan ichtyologique, sa faune piscicole est assez diversifiée et comprend 19 familles, 31 genres et espèces (INERA, non publié). Les principales espèces d'intérêt économique et alimentaire sont représentées par Heterotis niloticus (35\% des captures), tilapias $(32,5 \%)$, silures $(17 \%)$, synodontes $(6 \%)$, et des espèces «nobles » comme le Gymnarchus qui peut atteindre 17 à $20 \mathrm{~kg}$ (Baijot et al., 1994b). Les quatre espèces choisies dans cette étude ont une contribution pondérale très significative $(55 \%)$ dans les prises de cette pêcherie.

Le but de cette étude est d'établir et de donner des informations sur les paramètres de la relation poids-longueur chez les quatre espèces dominantes de la rivière Sourou, dans la région Nord-Ouest du Burkina.

\section{MATERIEL ET METHODES}

La collecte des données de longueur et de poids a duré 15 mois (septembre 2003 à novembre 2004), avec une périodicité de cinq jours correspondant à la fréquence des marchés dans la vallée du Sourou.

Les mensurations ont concerné les 4 espèces de poissons les plus abondantes et d' intérêt commercial capturées par les pêcheurs avec des filets à mailles $(35-40 \mathrm{~mm})$ et des hameçons appâtés de taille $\mathrm{n}^{\circ} 7$ à 12 pour le Gymnarchus (carnivore). Les mensurations de la taille ont été effectuées à $0,1 \mathrm{~cm}$ près. Chez Heterotis niloticus et Clarias gariepinus, nous avons mesuré la distance horizontale de l'extrémité antérieure du museau à la base (ou articulation) de la nageoire caudale . Chez Protopterus annectens, cette mesure a été effectuée de l'extrémité du museau jusqu'au point de jonction des nageoires dorsale et anale. Quant à Gymnarchus niloticus, la mesure est faite de l'extrémité du museau à la pointe de la caudale. Le poids total de chaque poisson a été mesuré au gramme près.

La relation entre la longueur des poissons et leur poids est représentée par la relation (Le Cren, 1951) : $\mathrm{W}=\mathrm{a} \mathrm{L}^{\mathrm{b}}$, où $\mathrm{W}$ est le poids total du poisson en $\mathrm{g}$ et $\mathrm{L}$ est la longueur totale du poisson en $\mathrm{cm}$; $\mathrm{a}$ et $\mathrm{b}$ sont des facteurs caractéristiques du milieu et de l'espèce. La transformation logarithmique linéaire de type $\ln (\mathbf{W})=\ln (\mathbf{a})+\mathbf{b} . \ln (\mathbf{L})$ permet de réduire la variabilité et d'homogénéiser les deux variables (W et L). La constante b est déduite de la droite de régression linéaire et varie entre 2 et 4 , mais il est souvent proche de 3 . La constante de proportionnalité a de la relation entre le poids et une puissance de la longueur était déterminée par calcul $\left(\mathbf{a}=\mathrm{e}^{\mathrm{x}}\right.$, avec $\mathbf{x}$ comme constante logarithmique de la courbe de régression) (Pauly et Moreau, 1997). La corrélation linéaire entre ces deux variables (poids et longueur) est exprimée par un coefficient $\left(\mathrm{r}^{2}\right)$. Dans le souci de vérifier si la valeur de $\mathbf{b}$ déduite des courbes de régression était différente de 3, nous avons employé le test de Student au seuil de signification de 5\%. Les paramètres de régression ont été calculés et analysés à l'aide de la version $14.0 \mathrm{du}$ logiciel SPSS.

\section{RESULTATS}

Les paramètres $\mathbf{a}$ et $\mathbf{b}$ de la relation longueur-poids de quatre espèces de poissons dominants de la rivière Sourou au Burkina Faso sont donnés au tableau 2. Les courbes de régression pour chacune des espèces examinées sont indiquées sur les figures $2 \mathrm{a}$, $2 \mathrm{~b}, 2 \mathrm{c}$ et $2 \mathrm{~d}$. Toutes les régressions étaient hautement significatives, avec le coefficient de détermination $\left(\mathrm{r}^{2}\right)$ variant de 0,765 à 0,947 $(\mathrm{p}<0,001)$. Les valeurs estimées de b pour Gymnarchus niloticus et Protopterus annectens n'étaient pas significativement différentes de $3(\mathrm{p}<0,001)$, traduisant ainsi pour ces deux espèces, une croissance de type isométrique. Pour Clarias gariepinnus la valeur de b était supérieure à $3(\mathrm{p}<0,001)$, traduisant ainsi une croissance allométrique positive. Quant à Heterotis niloticus, la valeur estimée de b était inférieure à $3(\mathrm{p}<0,001)$, traduisant ainsi une croissance allométrique négative.

\section{DISCUSSION}

Cette étude s'intéresse pour la première fois à la biologie de la croissance chez les poissons de la rivière Sourou, à travers l'étude de la relation longueur-poids de quatre espèces. Elle a permis de déterminer les 
Tableau 2: Paramètres de la relation longueur -poids et type de croissance de quatre espèces de poissons de la rivière Sourou, au Burkina Faso.

\begin{tabular}{|c|c|c|c|c|c|c|c|c|c|c|}
\hline \multirow{2}{*}{ Espèces } & \multirow[t]{2}{*}{$\mathbf{N}$} & \multicolumn{2}{|c|}{$\mathbf{L}$} & \multicolumn{2}{|r|}{$\mathbf{W}$} & \multirow[b]{2}{*}{$\mathbf{a}$} & \multirow{2}{*}{ b } & \multirow{2}{*}{$\mathbf{r}^{2}$} & \multirow{2}{*}{ SE } & \multirow{2}{*}{ Type de croissance } \\
\hline & & $\min$ & $\max$ & $\min$ & $\max$ & & & & & \\
\hline Heterotis niloticus & 294 & 22,3 & 67,1 & 200,2 & 3300,1 & 0,0344 & 2,70 & 0,765 & 0,087 & Allométrie négative \\
\hline Protopterus annectens & 300 & 23,2 & 99,3 & 100,1 & 3500,0 & 0,0052 & 2,95 & 0,853 & 0,060 & Isométrie \\
\hline Gymnarchus niloticus & 190 & 32,5 & 161,0 & 100,3 & 16000,0 & 0,0013 & 3,15 & 0,870 & 0,092 & Isométrie \\
\hline Clarias gariepinus & 187 & 23,1 & 70,3 & 100,0 & 4400,2 & 0,0059 & 3,14 & 0,947 & 0,054 & Allométrie positive \\
\hline
\end{tabular}

$\mathrm{N}=$ Effectif (nombre de poissons examinés)

$\mathrm{W}=$ Poids vif exprimé en $\mathrm{g}$

$\mathrm{L}=$ Longueur standard exprimée en $\mathrm{cm}$

$\mathrm{SE}=$ Erreur Standard 


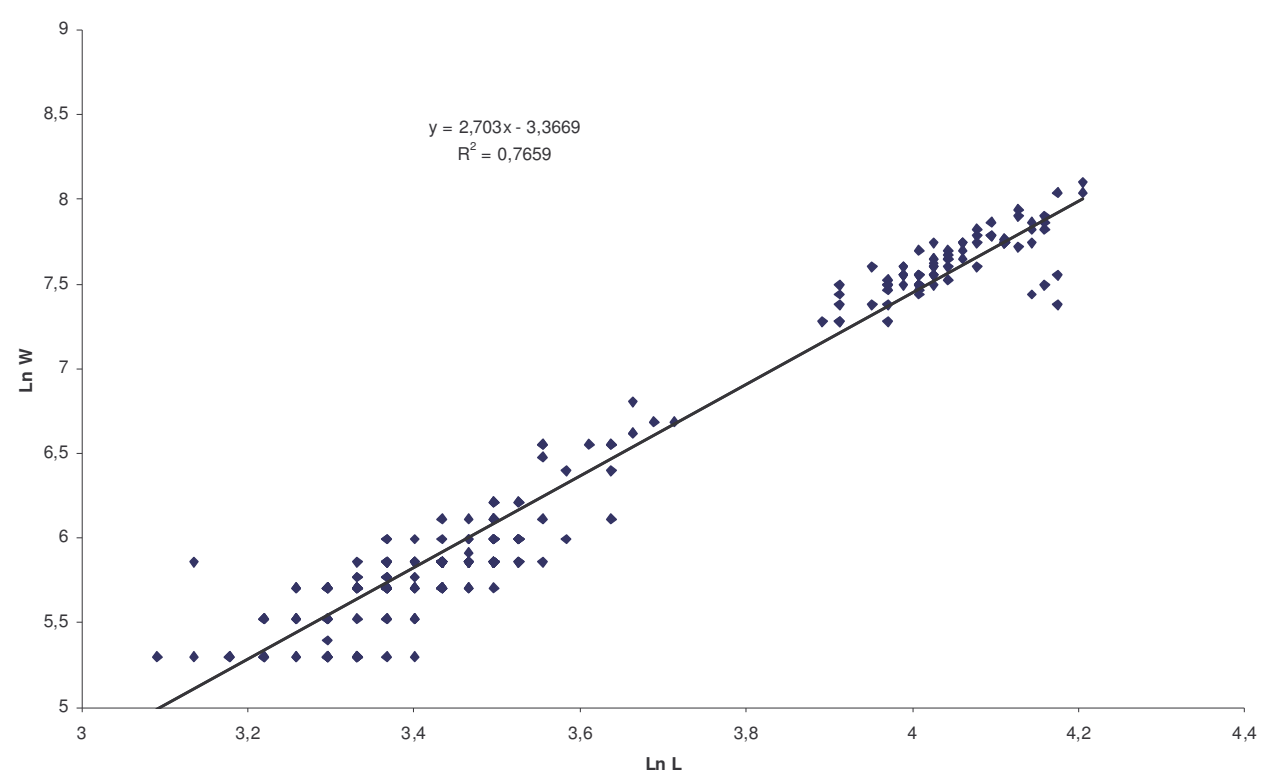

Figure 2a: Courbe Longueur-Poids de Heterolis niloticus du fleuve Sourou ( $\mathrm{n}=294)$.

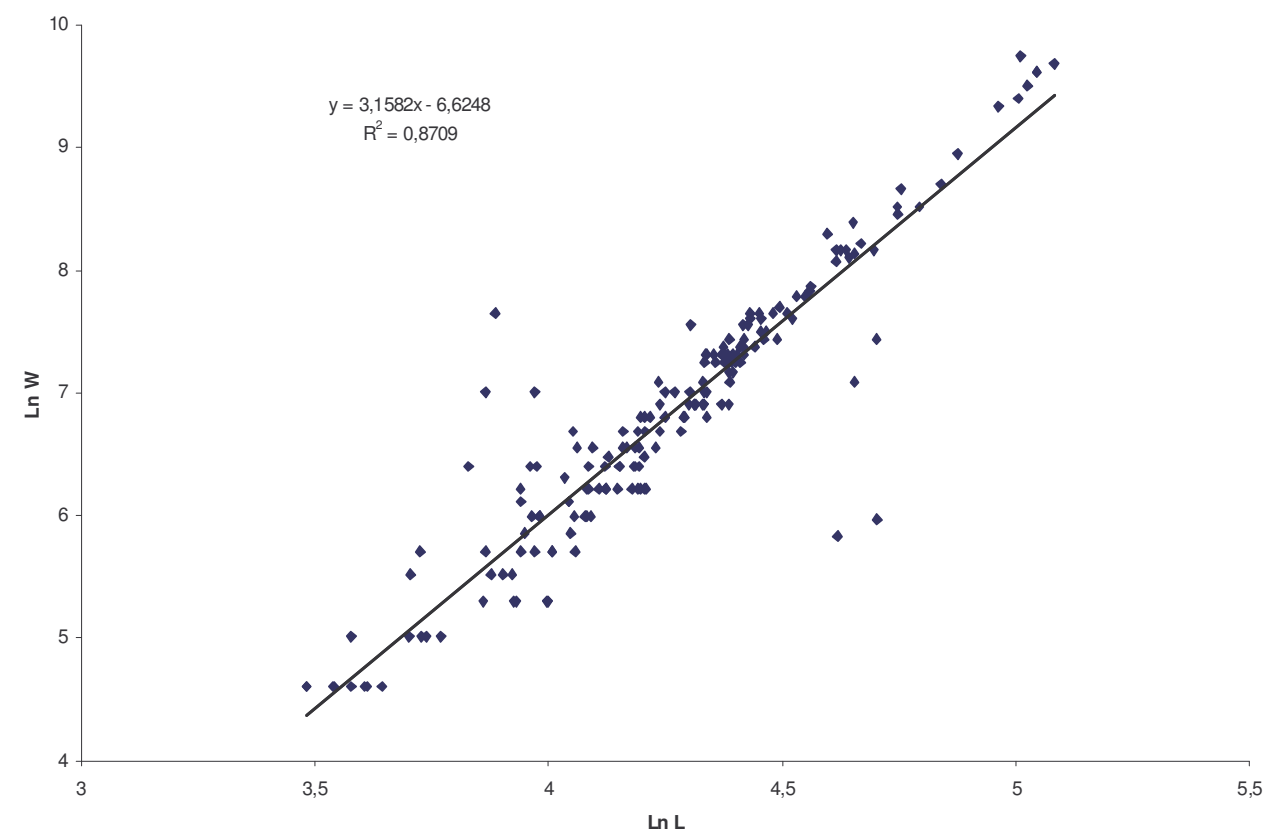

Figure 2b: Courbe Longueur-Poids de Gymnarchus niloticus du fleuve Sourou ( $\mathrm{n}=190)$.

paramètres « $\mathbf{a}$ et $\mathbf{b} »$ de la relation. Dans l'ensemble la valeur de « $\mathbf{b} »$ a varié de 2,70 à 3,15 . Cette fourchette de la valeur de $\langle\mathbf{b} »$ est concordante avec celle habituellement rapportée et admise par la littérature et qui situait cette valeur entre 2,50 et 3,50 (Pauly et Moreau, 1997). 


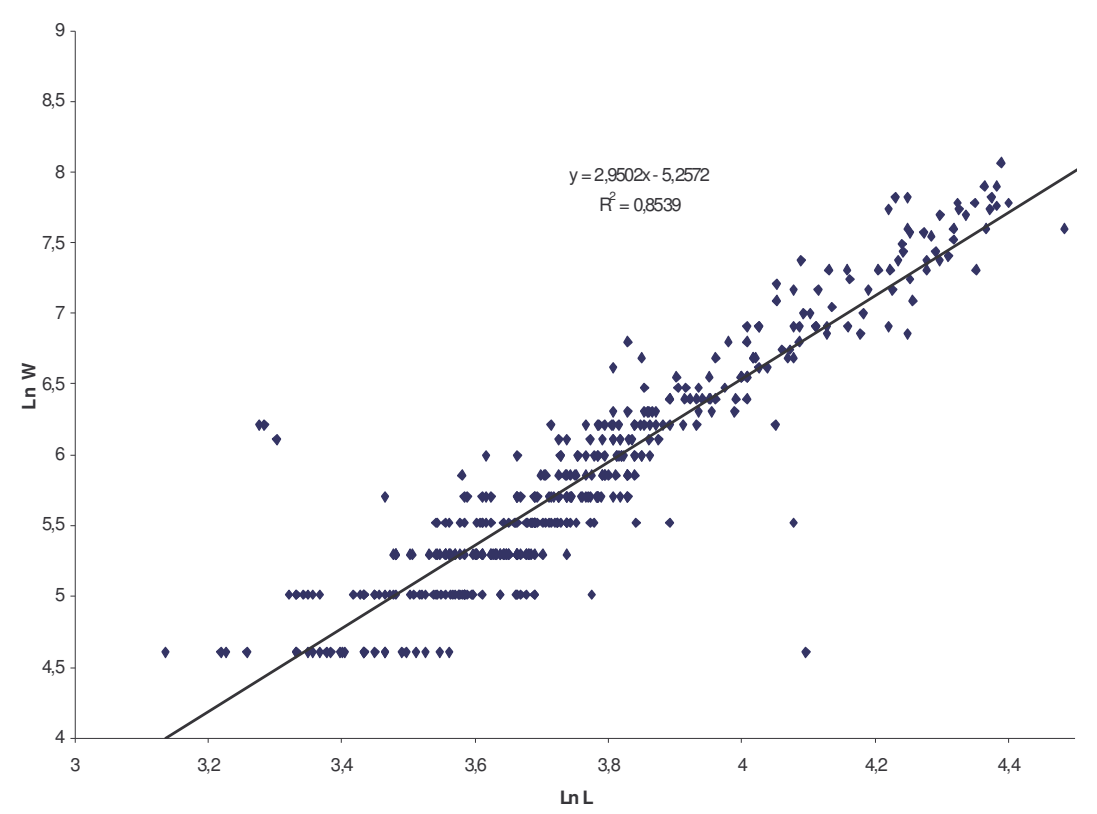

Figure 2c: Courbe Longueur-Poids de Protopterus annectens du fleuve Sourou $(\mathrm{n}=300)$.

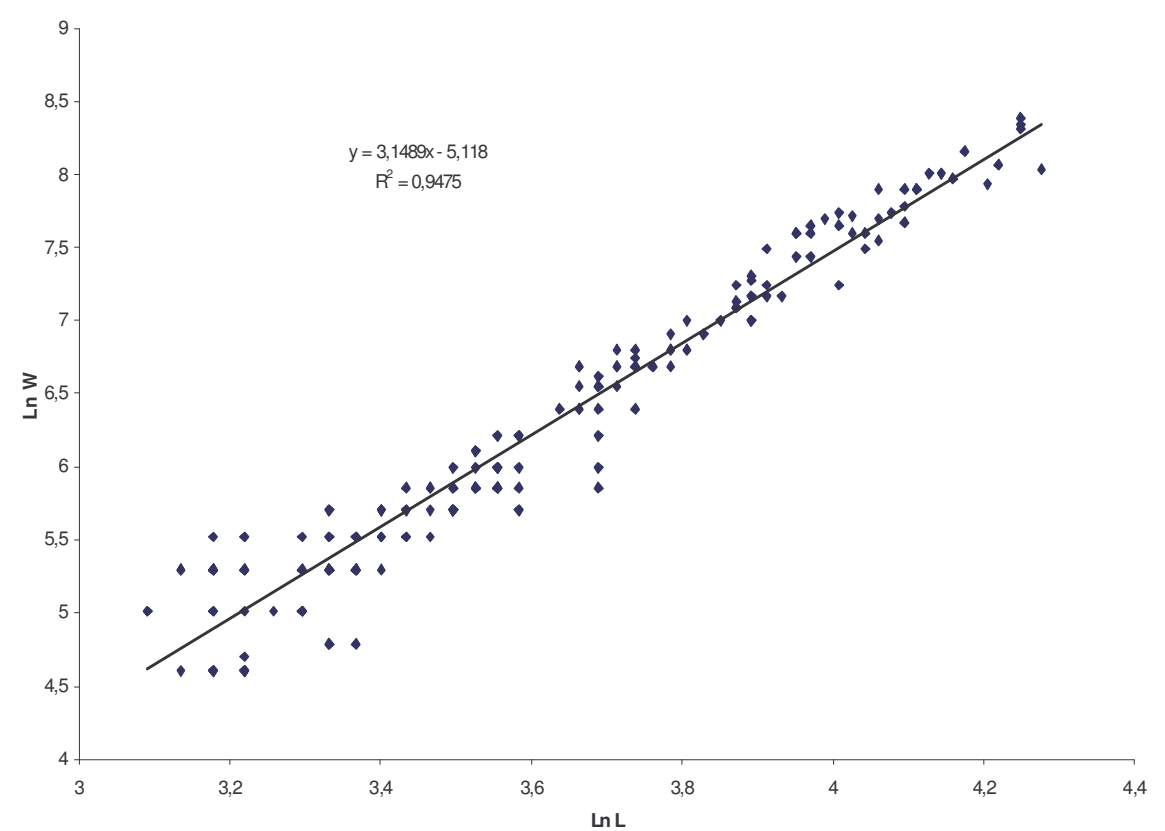

Figure 2d: Courbe Longueur-Poids de Clarias gariepinus du fleuve Sourou (n=187).

L'examen de cette valeur de b fait distinguer chez les poissons étudiés, trois types de croissance. En effet, chez un poisson idéal qui maintient en parfaite égalité les dimensions du corps et la longueur, la valeur isométrique de b est de 3. Cela est observé dans notre étude chez Protopterus annectens et Gymnarchus niloticus. Chez ces deux espèces, la forme du corps ne change pas avec la croissance. En réalité, des déviations de cette croissance de type isométrique sont souvent observées car de nombreux poissons 
changent la forme de leur corps au cours de leur croissance. Aussi, une valeur de b inférieure à 3 (cas de Heterotis niloticus) indique que le poisson devient plus mince au cours de sa croissance en longueur. Il s'agit d'une croissance allométrique négative où le développement du corps (croissance pondérale) est moins rapide que celui de la longueur. A l'inverse, dans le cas d'une croissance allométrique positive, la valeur de b est supérieure à 3 (cas de Clarias gariepinnus) et dénote que le poisson prend de l'embonpoint au cours de sa croissance en longueur.

En comparaison, la valeur de $\mathbf{b}(3,14)$ chez Clarias gariepinus au Sourou, est presque égale à celles observées à la Mare aux Hippopotames $(3,21)$, à la vallée de la rivière Kou (3, 09), sur le fleuve Nakambé $(3,20)$ et les petites pêcheries (Ramitenga, Tanguiga, Boulmiougou, Kokologho) du Centre $(3,23)$ d'après Baijot et al. (1994c), Sanon (1995) et Coulibaly (2003). Pour la même espèce, cette valeur était supérieure à celle rapportée par Hickley et Bailey (1986) au Soudan sur le fleuve Nil $(2,75)$ et par le GPSO (1995) dans le sud-ouest du Burkina $(2,95)$ sur six petites pêcheries: Tounoura, Comoé, Tiéfora, Douna, Lemourdougou et Navrikpê. Sur la rivière Idodo au Nigéria, Anibeze (2000) a trouvé une valeur de b $(2,15)$ inférieure chez Heterobranchus longifilis, une espèce proche de Clarias gariepinus.

Chez Heterotis niloticus, la valeur de b $(2,70)$ est inférieure à 3 dans les pêcheries de la Mare aux Hippopotames (Bearez, 2003), de la Vallée de la rivière Kou (Sanon, 1995) et du fleuve Nakambé (Coulibaly, 2003). Sur le fleuve Nil au Soudan, Hickley et Bailey (1986) ont rapporté par contre une valeur de « $\mathbf{b} »$ de 3,08 pour la même espèce.

Les données relatives à la croissance des poissons dans les écosystèmes lotiques sahéliens, comme celui de la rivière Sourou, sont très peu abondantes. Ces écosystèmes représentent un important support de la production piscicole. Un suivi régulier des captures et de l'activité de pêche (effort de pêche) permettront de mieux apprécier dans les années à venir, les effets de la pêche extensive sur la croissance de ces poissons à travers l'analyse des paramètres de la relation longueur-poids. D'autres paramètres comme le facteur de condition pourront être établis et permettre la comparaison du «bien - être » de ces populations de poisson dans le même milieu.

\section{BIBLIOGRAPHIE}

Anibeze CIP. 2000. Length-weight relationship and relative condition of Heterobranchus longifilis (Valenciennes) from Idodo River, Nigeria. Naga, 23(2): $34-35$.

Baijot E, Bouda S, Ouédraogo L. 1994a. Etude des conditions physico-chimiques et biologiques des retenues d'eau du Burkina Faso. In Aspects Hydrobiologiques et Piscicoles des Retenues d'Eau en Zones Soudano-sahélienne, Baijot E, Moreau J, Bouda S (eds). CTA-CEE: Bruxelles; 37-64.

Baijot E, Kaboré K, Zerbo H. 1994b. Production exploitée et effort de pêche dans les retenues d'eau. In Aspects Hydrobiologiques et Piscicoles des Retenues d'Eau en Zones Soudanosahélienne, Baijot E, Moreau J, Bouda S (eds). CTA-CEE : Bruxelles ; 123-157.

Baijot E, Barry I, Ratjs F. 1994c. Peuplements piscicoles des retenues du Burkina Faso. In Aspects Hydrobiologiques et Piscicoles des Retenues d'Eau en Zones Soudanosahélienne, Baijot E, Moreau J, Bouda S (eds). CTA-CEE : Bruxelles ; 65 - 85.

Béarez P. 2003. La mare aux hippopotames (Burkina Faso): aspects hydrobiologiques et halieutiques. In Fish Biology: Local Studies as Basis for Global Inferences, Palomares MLD, Samb B, Diouf T, Vakily JM, Pauly D (eds). ACPUE Fisheries Research Report $\mathrm{n}^{\circ} 14$ : Bruxelles ; 98 - 107.

Coulibaly ND. 2003. Relation taille-poids de 11 espèces de poisons du Burkina Faso. In Fish Biology: Local Studies as Basis for Global Inferences, Palomares MLD, Samb B, Diouf T, Vakily JM, et Pauly D (eds). ACP-UE Fisheries Research Report $\mathrm{n}^{\circ} 14$ : Bruxelles ; $20-22$.

Coulibaly ND. 2006. Pêcherie $d u$ Sourou; Fiche Technique. INERA/DPF: Ouagadougou.

Dankwa HR. 2003. Biology of Polypterus senegalus (Pisces, Polypteridae) in the Pru River, Ghana. In Fish Biology: Local Studies as Basis for Global Inferences. 
Palomares MLD, Samb B, Diouf T, Vakily JM, Pauly D (eds). ACP-UE Fisheries Research Report $\mathrm{n}^{\circ} 14$ : Bruxelles; 23 - 24.

Durand JR, Franc J, Loubens G. 1973. Clefs Longueurs-poids pour 58 Espèces de Poissons du Bassin du Lac Tchad. ORSTOM : Fort-Lamy.

Frota LO, Costa PAS, Braga AC. 2004. Length-weight relationship of marine fishes from the central Brazilian coast. Naga, 27(1-2): 21 -26.

GPSO (Projet Gestion des Pêches dans le SudOuest). 1995. Enquêtes Halieutiques sur les Six Retenues: Tounoura, Comoé, Douna, Tiéfora, Lemouroudougou et Navrikpê. Situation et Recommandations. GOPA-COFAD: Bad Homburg.

Harbott BJ, Ogari JTN. 1982. The biology of the larger cichlid fish of lake Turkana. In Lake Turkana. a Report of the Findings of the Lake Turkana Project 1972 - 1975, Hopson AJ (ed.). Overseas Development Administration : London ; 1331 - 1355.

Hickley P, Bailey RG. 1986. Fish communities in the perennial wetland of the Sudd, southern Sudan. Fresh. Biol., 16: $695-709$.

Kochzius M. 1997. Length-weight relationships of fishes from a seagrass meadow in Negros Oriental, Philippines. Naga, 2(3-4): 64 - 65.

Le Cren ED. 1951. The length-weight relationships and seasonal cycle in gonad weight and condition in perch (Perca fluviatilis). Journal of Animal Ecology, 20(2): $201-219$.

Le Tourneur Y, Kulbicki M, Labrosse P. 1998. Length-weight relationships of fishes from coral reefs and lagons of New Caledonia - an update. Naga, 21(4): 39-
46.

Lévêque C. 1999. Croissance et ontogénie. In Les Poissons des Eaux Continentales Africaines: Diversité, Ecologie, Utilisation par l'Homme, Lévêque C, Paugy D (eds). IRD : Paris (France); 153166.

Loubens G. 1974. Quelques aspects de la biologie des Lates niloticus du Tchad. Cahiers ORSTOM, sér. Hydrobiol., 8: 321.

Pauly D, Moreau J, 1997. Méthodes pour l'Evaluation des Ressources Halieutiques. CEPADUES: Toulouse.

Odat N. 2003. Length-weight relationship of fishes from coral reefs along the coastline of Jordan (Golf of Aqaba). Naga, 26(1): 9-10.

Ruiz-Ramirez S, Lucano-Ramirez G, Mariscal-Romero J, 1997. Length-weight relationships of soft-bottom demersal fishes from Jalisco and Colima States, Mexico. Naga, 20(3-4): 62-63.

Sanon ZL. 1995. Inventaire et dynamique de quelques espèces de poissons dans la Réserve de la Biosphère de la mare aux hippopotames et dans le lac de la vallée du Kou. Mem. Ingéniorat, Université/Inst. Dévelop. Rural de Ouagadougou, Ouagadougou, p.59.

Stergiou KI, Moutopoulos DK. 2001. A review of length-weight relationship of fishes from Greek Marine Waters. Naga, 24 (1-2): 23-39.

Thomas J. Venu S, Kurup B.M. 2003. Lengthweight relationship of some deep-sea fish inhabiting the continental slope beyond $250 \mathrm{~m}$ along the west coast of India. Naga, 26(2): 17-21. 